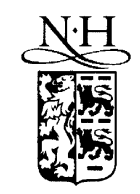

ELSEVIER
Available at

www.ComputerScienceWeb.com

powered by science dDirecto

The Journal of Systems and Software xxx (2003) xxx-xxx

\title{
Roam, a seamless application framework
}

\author{
Hao-hua Chu *, Henry Song, Candy Wong, Shoji Kurakake, Masaji Katagiri \\ DoCoMo Communications Laboratories USA, Inc., 181 Metro Drive, Suite 300, San Jose, CA 95110 USA \\ Received 23 December 2002; accepted 28 December 2002
}

6 Abstract

One of the biggest challenges in future application development is device heterogeneity. In the future, we expect to see a rich

8 variety of computing devices that can run applications. These devices have different capabilities in processors, memory, networking,

9 screen sizes, input methods, and software libraries. We also expect that future users are likely to own many types of devices. Depending on users' changing situations and environments, they may choose to switch from one type of device to another that brings the best combination of application functionality and device mobility (size, weight, etc.). Based on this scenario, we have designed and implemented a seamless application framework called the Roam system that can both assist developers to build multi-platform applications that can run on heterogeneous devices and allow a user to move/migrate a running application among heterogeneous devices in an effortless manner. The Roam system is based on partitioning of an application into components and it automatically selects the most appropriate adaptation strategy at the component level for a target platform. To evaluate our system, we have created several multi-platform Roam applications including a Chess game, a Connect4 game, and a shopping aid application. We also provide measurements on application performance and describe our experience with application development in the Roam system. Our experience shows that it is relatively easy to port existing applications to the Roam system and runtime application migration latency is within a few seconds and acceptable to most non-real-time applications.

\section{Introduction}

The era of PC-dominated applications is about to end. Now-a-days, we see widespread use of mobile devices that have sufficient computing and networking capabilities to run a variety of feature-rich applications. They come in a variety of form factors, including smart pagers, cell phones, PDAs (Pocket PCs), handheld PCs, car navigation systems, and notebook PCs. An average person may already own multiple such devices. It is expected that in the near future, the number of such devices will far exceed the number of desktop PCs. As a consequence we expect that the predominant software platform will shift from PCs to mobile devices. This creates a challenge for developers to build applications that can run on different mobile device platforms. To address this challenge, we believe that there is a need to

\footnotetext{
${ }^{*}$ Corresponding author.

E-mail addresses: haochu@docomolabs-usa.com (H.-h. Chu), csyus@docomolabs-usa.com (H. Song), wong@docomolabs-usa.com (C. Wong), kurakake@docomolabs-usa.com (S. Kurakake), katagiri@docomolabs-usa.com (M. Katagiri).
}

provide an application framework for building multiplatform mobile applications.

We also expect a mobile user may choose to switch from one type of device to another type of device, in the middle of using an application, in order to access necessary application functionality or to become more mobile, based on changing situations, environments, or needs. For example, a user starts planning a vacation online using a desktop computer in his/her office. In the middle of planning, he/she receives an urgent call and must leave the office for a meeting at a remote site. The user would like to continue planning the trip on the bus or during break time between meetings. Given the need for mobility, he/she switches to a lightweight, mobile device (PDA or cell phone) away from the office. Based on this scenario, we believe that when mobile users switch devices, there is a need to allow them to move any running application effortlessly between devices.

Based on these two needs, developers will be required to write seamless applications. We define a seamless application to be an application that can run on heterogeneous devices and migrate at runtime among heter- 
60 ogeneous devices. There has been an abundance of re61 search in the area of mobile agents that allow applica62 tions to move from one host to another at runtime with 63 little or no loss of execution states (Aramira, 1999; 64 Acharya et al., 1997; Banavar et al., 2000; Fünfrocken, 65 1998; Gray et al., 1997; Lange and Oshima, 1998; Mil66 ojicicc et al., 1998; Mitsubishi Electric ITA Horizon 67 Systems Laboratory, 1998; ObjectSpace, xxxx; Peine 68 and Stolpmann, 1997; Strasser et al., 1996; White et al., 69 1997). These systems are mostly built on top of the Java 70 virtual machine (JVM), which provides the advantage of 71 a common runtime environment. However, these sys72 tems make an important assumption of device homoge73 neity. For example, the underlying hosts/devices must be 74 PC or PC-like devices with enough hardware/software 75 (HW/SW) capabilities to run the standard JVM. Given 76 this device homogeneity assumption and Java's "Write 77 Once, Run Anywhere" programming model, it is rela78 tively straightforward to realize runtime application 79 migration among similar devices. However, device ho80 mogeneity is not a realistic assumption in today's mobile 81 computing environment where there exists a wide range 82 of mobile devices and information appliances with dif83 ferent HW/SW capabilities. For an example, a cell 84 phone or a PDA in comparison to a desktop computer 85 has a slower processing speed, less memory, little or no 86 permanent storage, slower and unreliable network con87 nectivity, smaller screen size, and limited input capa88 bilities. To address device heterogeneity, Java introduces 89 mutually incompatible Java 2 Micro Edition (J2ME) 90 profiles and configurations for different classes of mobile 91 devices. As a result, Java's "Run Anywhere" ideal does 92 not apply to the device heterogeneous environment.

\section{1.1. Challenges}

The focus of the Roam system is to address the device heterogeneity problem in runtime application migration. Fig. 1 shows different aspects of this problem that a Java application developer may face when developing a seamless application.

- Incompatible Java virtual machine configurations and profiles: Java provides incompatible virtual machine (VM) configurations and profiles for different classes of mobile device. These VM configurations and profiles support different subsets of APIs that application developers can use to build applications. A device with more capable HW (e.g., more memory and faster processors) can typically support a VM with a more extensive Java class library. If an application uses APIs provided by a Java profile or configuration of a more capable platform, this application may not be able to run on less capable platforms that do not support these APIs.

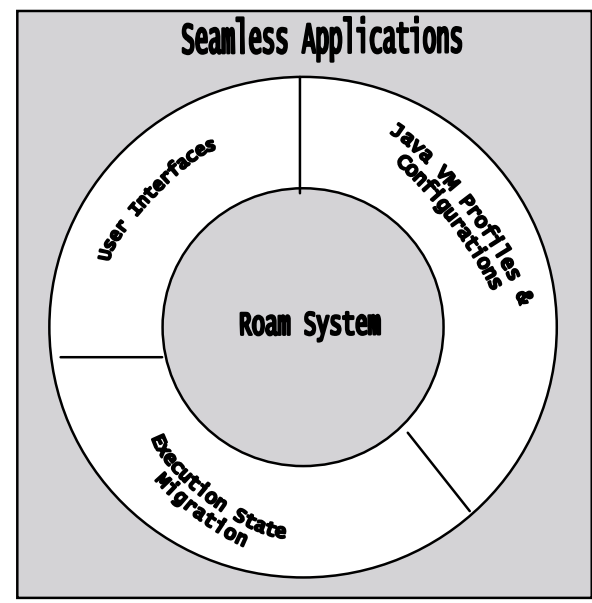

Fig. 1. Challenges for application migration in heterogeneous device environment.

- Execution state migration: In a runtime application 112 migration from a source device to a target device, 113 the execution state of an application needs to be cap- 114 tured on the source device, transferred to a target de- 115 vice and restored. The application execution state 116 includes heap, stack, network sockets, file I/O state, 117 and other state information. Device heterogeneity 118 brings an additional challenge in execution state mi- 119 gration. Given the incompatible configurations and 120 profiles on different device platforms, application de- 121 velopers may choose to create multiple device-depen- 122 dent implementations (M-DD) for an application 123 component (e.g., one implementation for each spe- 124 cific device platform), or they can use an automated 125 tool that can transform an application component 126 into code that can be run on each platform. This 127 means that, if an application has two different imple- 128 mentations (either hand-coded or transformed) on 129 two different platforms, the captured runtime execu- 130 tion state on the source device may not correspond 131 to the different implementation on the target device. 132

- User interface: Different platforms have different dis- 133 play sizes and input methods. A DoCoMo 503i cell 134 phone has an approximately $120 \times 130$ pixel display 135 and a small numeric keypad; a Compaq iPaq Pock- 136 etPC has a $320 \times 240$ pixel display and a stylus; and 137 an average notebook PC has a $1024 \times 768$ pixel dis- 138 play, a keyboard and a pointing device. Display size 139 can change the presentation and layout of the GUI 140 components. A large display (e.g., desktop PC) can 141 accommodate big GUI components, and many 142 GUI components can be presented at once in the 143 same window or page. On the other hand, a small dis- 144 play can only accommodate highly compact GUI 145 components, and only a few GUI components can 146 be displayed at once. In addition, the constraints of 147 the devices' display sizes and input methods can affect 148 
the range of tasks that are appropriate on each platform. For example, a notebook PC or a Blackberry pager with a keyboard is suitable for performing tasks that involve text entry. On the other hand, a cell phone with only a numeric keypad is prohibitively difficult to use for tasks involving text entry.

\subsection{General approach}

To address the challenges described in the previous section, the Roam system provides a seamless application framework for developers to build resource-aware seamless applications. By resource-aware, we mean that applications are aware of the underlying device capabilities (Java VM configuration and profile, display size, input method, memory, network, etc.), which may change when they migrate between devices. In order to migrate applications between devices with different capabilities, adaptation is needed. The Roam system utilizes the following three adaptation strategies:

- Dynamic instantiation: An application is divided into M-DD components that perform the same function, but each component is implemented/re-implemented for different platforms. At migration time or runtime, the set of components that best fit the target device capabilities is selected for instantiation. For example, the Java VM configuration and profile supported on the target device is considered as a part of the target device capabilities in the Roam system. A developer can provide two device-dependent implementations of the same component, one for cell phones that support the J2ME DoCoMo Java (DoJa) profile, and another for notebook PCs that support the Java 2 Standard Edition (J2SE) VM. When an application is started on or migrated to a cell phone, the Roam system dynamically instantiates the cell phone implementation.

- Offloading computation: The Roam system allows applications to make use of distributed computing resources to run software components that are beyond the target device capabilities. These components may require a more capable Java VM, or have certain CPU, memory, or network requirements, such that it is necessary to dispatch them to remote servers that can better support these requirements. Consider an application that has a component implemented to run only on the J2SE VM. When this application is started on or migrated to a cell phone, the Roam system finds a remote server capable of running the J2SE $\mathrm{VM}$ and offloads that software component to that remote server.

- Transformation: The Roam system allows device-independent components to be transformed at runtime to fit the target device capabilities. It provides a device-independent GUI toolkit that a developer can use to build user interface (UI) components. At migration time or runtime, these device-independent UI components are transformed to run on the target device.

The Roam system is a framework to assist developers in building applications that can adapt to heterogeneous devices. At design time, a developer must make the decision on how to partition an application into separate components, and for each component, whether to provide multiple device-dependent implementations (M$\mathrm{DD})$, a single device-dependent implementation (S-DD), or a single device-independent presentation (S-DI). At runtime or migration time, the Roam system applies the most appropriate adaptation strategy, shown in Table 1, based on the developer's design decision and the target device capabilities.

We believe that some adaptation strategies are better suited for certain types of application components. For some UI components, we believe that transformation adaptation may be the most appropriate, given that most UI components cannot be offloaded to a remote server for execution. However, device-independent UI authoring sometimes does not result in high-quality interfaces. In these cases, M-DD UI components are developed, and dynamic instantiation adaptation is preferred. This decision is up to the UI developer. For application logic components, we believe that S-DD implementation, using offloading computation adaptation is often most suitable, because most application logic components can be offloaded to remote servers for execution.

In order to suspend and resume the execution of mobile applications across heterogeneous devices, the Roam system must be able to capture execution state on the source device, serialize and transfer it to the target device, and then restore it. Because M-DD components may be dynamically instantiated, and the implementation of each is different, execution state must be captured using a device-independent state representation,
194 195 196 197 201 202 203 204 205

Table 1

Summary of adaptation strategies

\begin{tabular}{lll}
\hline Adaptation strategies & Application components & Intended for \\
\hline Dynamic instantiation & Multiple device-dependent implementations (M-DD) & Customized, high-quality UI components \\
Offloading computation & Single device-dependent implementation (S-DD) & Application logic components \\
Transformation & Single device-independent presentation (S-DI) & UI components \\
\hline
\end{tabular}


241 and converted into representations specific to each 242 component implementation using execution state trans243 formation. The Roam system requires developers to 244 provide execution state transformation logic for M-DD 245 components. There is no need for a developer to provide 246 execution state transformations for S-DI or S-DD 247 components that have a single implementation. When 248 Roam system applies transformation adaptation to an 249 S-DI component, it automatically generates the corre250 sponding execution state transformation.

251 Another important requirement is Security. Proper 252 security policy must be enforced to ensure that only 253 authorized applications are allowed to migrate from one 254 device to another.

\section{Design} as follows:
The Roam system architecture is shown in Fig. 2. A Roamlet is an application that runs in the Roam system. A Roamlet can migrate between any two connected devices that have the Roam system running. The architecture contains three main components: Roam Agent, Roamlet, and HTTP Server. Roam agents must be installed and executed on both the source and target devices before a Roamlet can run, and before any Roamlet migration can occur. The flow for a Roamlet migration from a PC device to a PDA device is described

1. The Roam agent on the source device first negotiates with the Roam agent on the target device. The negotiation involves exchanges of the target device capabilities, the device capabilities needed by each application component, and the code base URL where the Roamlet component byte code can be downloaded from. Based on the exchanged information, the Roam agent decides the appropriate adaptation strategy for each component.

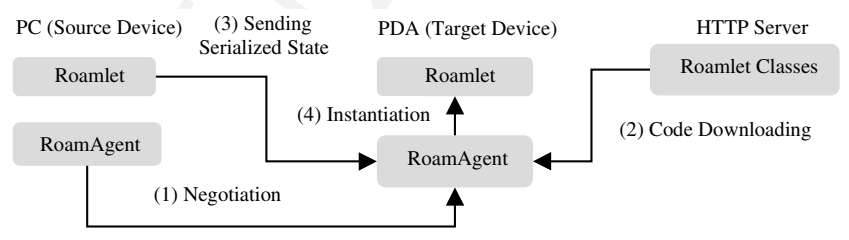

Fig. 2. Roam system architecture.
2. The Roam agent on the target device downloads the necessary Roamlet class byte code from the HTTP server for all application components that will be instantiated on the target device.

3. The Roamlet on the source device serializes its execution state and sends it to the Roam agent on the target device. The Roam agent may perform execution state transformation if an application component is transformed or dynamically instantiated.

4. The Roam agent instantiates the Roamlet on the target device.

Roamlets are based on a component-based programming model: they are built as components that can be distributed across multiple devices, and can be composed together into a working application through welldefined interfaces. Fig. 3 shows an example of a Roamlet partitioned into three components: a GUI component with device-independent representation (SDI), a second GUI component with two device-dependent implementations on PC and PDA (M-DD), and an application logic component with a PC implementation (S-DD). The component-based programming model allows the Roam system to apply a different adaptation strategy on different application component, such as offloading an S-DD component to a remote device, dynamically instantiating an M-DD component of a different implementation, and transforming an S-DI component without affecting other components in a Roamlet. This requires each component to invoke procedure calls of any other components regardless of whether they are running on remote or local hosts. In our current Roam system, the component-based programming model is built on top of Java Remote Method Invocation (RMI).

Roamlets and Roam agents use resource specifications to describe the device requirements for each component implementation, and the capabilities of a host device, respectively. At migration or load time, the Roam agent can compare the device requirements from the application components with the target device capabilities and decide the best adaptation strategy for each application component. Note that components in a Roamlet may have different device requirements, so a Roamlet is required to provide a separate set of device requirements for each component implementation. The current Roam system supports specification of a limited set of device capabilities, including Java VM configuration or profile, display size and input method, as shown in Table 2. For example, device capabilities for a PC are $\{$ J2SE VM, $1024 \times 780$ pixels, keyboard and mouse $\}$. The device requirements for the PDA implementation of the GUI component shown in Fig. 3 can be $\{$ Personal Java VM, $320 \times 240$ pixels, stylus\}, and the PC implementation of the GUI component can be \{J2SE VM, $1024 \times 780$ pixels, keyboard and mouse\}. 


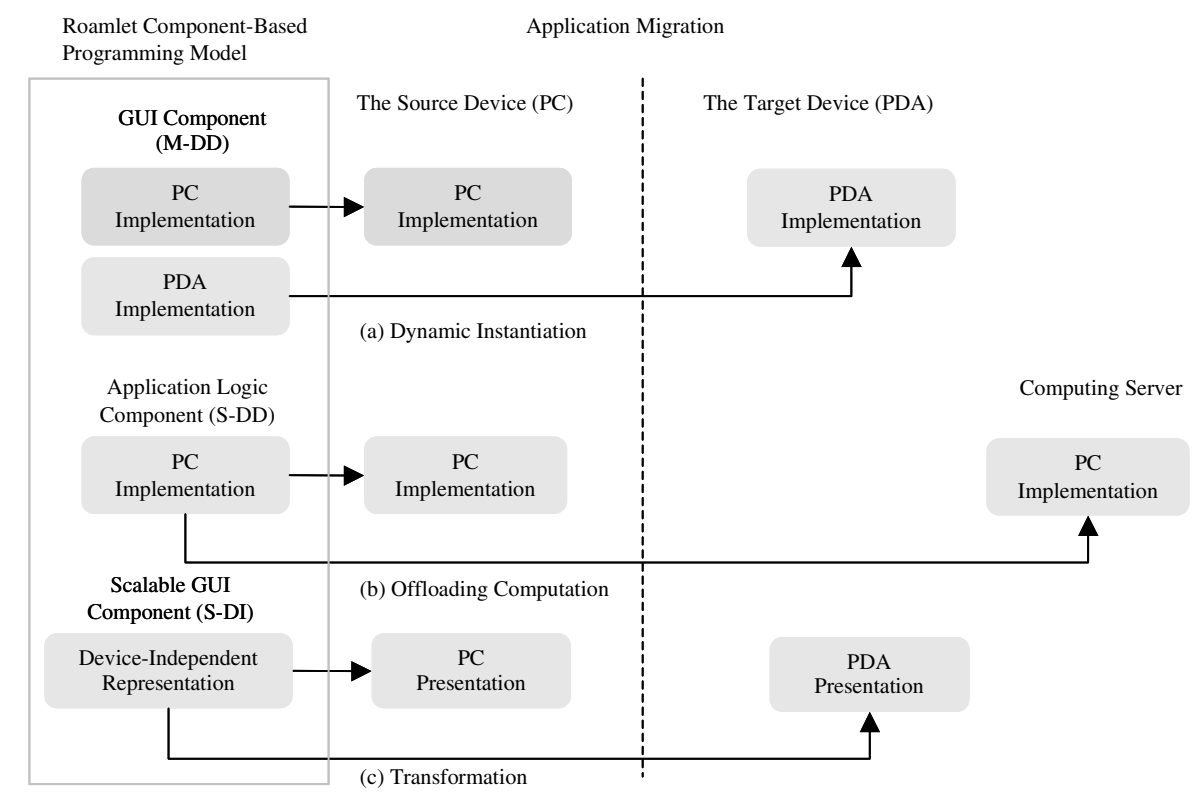

Fig. 3. Roamlet component-based programming model and different adaptation strategies for Roamlet components.

Table 2

Specification for device capabilities

\begin{tabular}{llcl}
\hline & Types of Java VM & Display sizes & Input methods \\
\hline PC device capability & J2SE & $1024 \times 780$ & Mouse and keyboard \\
Pocket PC device capability & PersonalJava & $320 \times 240$ & Stylus and virtual keyboard \\
Cell phone device capability & J2ME/DoJa profile & $100 \times 100$ & Keypad \\
\hline
\end{tabular}

337 Since the scalable GUI (SGUI) component has a device338 independent representation, its device requirements can 339 contain a range of capabilities.

\subsection{Dynamic instantiation}

In dynamic instantiation, a developer provides $\mathrm{M}$ DD implementations for a Roamlet component. The rule for selecting the most suitable device-dependent implementation is that the component implementation that best matches (but not exceeds) the target device capability is selected. An example of a Roamlet migrating from a PC to a PDA is shown in Fig. 3(a). The Roam agent finds that the PDA implementation of the second GUI component exactly matches the target device (PDA) capability and instantiates it.

Using dynamic instantiation, Roamlet developers can program different Roamlet behaviors in separate code segments. At migration time the Roam system loads only the code segment that exhibits a behavior suited for the target device capability. Note that dynamic instantiation is different from programming different behaviors in the same code segment, which is not always desirable in Java. For this approach to work, the Roamlet has to be programmed to run on the least capable device (the lowest-common denominator approach) because the code segment that runs on different VM configurations and devices can only assume the most primitive API libraries provided by the least capable VM and devices. This is obviously undesirable for application programmers who would like to take advantage of rich class libraries provided by more capable devices.

The Connect4 Roamlet is a multi-platform game that we implemented to illustrate dynamic instantiation adaptation. Its GUI component provides M-DD presentations, one matching the PDA device capability and one matching the PC device capability. When Connect4 is instantiated on the PC, its $\mathrm{PC}$ presentation is selected for instantiation (Fig. 4(a)). When the user later migrates the Connect4 game to a PDA, its PDA presentation is selected for instantiation (Fig. 4(b)). The PDA presentation is a scaled down version of the PC presentation with some features and buttons removed to fit the smaller PDA display size (such as "undo" and "new game").

Dynamic instantiation creates a challenging situation, where a Roamlet is instantiated with a different implementation on the source device from that on the target device, as shown in Fig. 3(a). The execution state of the PC GUI component on the source device does not correspond to the PDA GUI component instantiated on
361

362

363

364

365

366

367

368

369

370

371

372

373 
(a) PC Presentation



(b) PDA Presentation

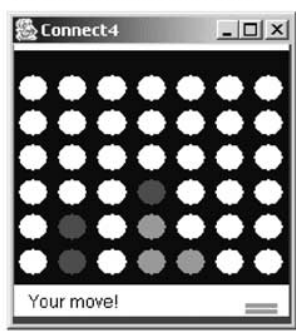

Fig. 4. Screenshots of M-DD presentations of Connect4 Roamlet: one presentation for PC and one presentation for PDA.

the target device. For example, the PC GUI component

\subsection{Offloading computation}

Offloading computation allows a Roamlet to delegate

398 the execution of computation- and memory-intensive 399 components to any remote server that has the device 400 capabilities to run them. Offloading computation is in401 tended for an application component that has a S-DD 402 implementation. Fig. 3(b) shows offloading computation 403 for an S-DD application logic component. After the 404 Roam agent finds that the application logic component 405 requires a PC-equivalent device, which exceeds the PDA 406 host's capabilities, it attempts to find a computing server 407 (PC) where it can offload this application logic compo408 nent. Both the Roamlet users and the Roam agent can 409 specify a list of servers that can accept offloadable 410 components. When a server is found, the Roam agent 411 directs the application logic component to be migrated 412 to that server. A Roam agent can apply offloading 413 computation to an M-DD component if none of its 414 device-dependent implementations can run on the target 415 device. For example, if an M-DD component has two 416 implementations on PC and PDA and the target device 417 is a cell phone, dynamic instantiation will fail. In this 418 case, the Roam agent will find the most capable server 419 on the list, and offload an appropriate M-DD compo420 nent to it. The precedence rule for applying different 421 adaptation strategies is that a Roam agent first applies dynamic instantiation adaptation to an application component. If the dynamic instantiation adaptation fails, it will apply offloading computation adaptation.

The Connect 4 game in Fig. 4 contains a S-DD application logic component that is offloadable. The application logic component keeps track of the state of the game and implements an artificial intelligence (AI) engine that computes the computer's next move. Since the AI could be computationally- and memory-intensive, it requires a device with a fast processor and sufficient memory. When the Connect4 game is running on the PC, the application logic component is instantiated locally. When the Connect4 game is migrated to the PDA, the application logic component is offloaded to a computing server.

Not all components in a Roamlet are offloadable. The Roam system allows a Roamlet to specify its components as either non-offloadable or offloadable components. Non-offloadable components are required to run on the target device. For example, GUI components and security-sensitive components are rarely offloaded. If the target device does not have the capability to run the non-offloadable components, the application migration simply fails and an error is presented to the user. A Roamlet can also specify components as reverseoffloadable or non-reverse-offloadable. Consider the same example in Fig. 3(b). At a later time, the user wants to migrate the Roamlet back to the PC from the PDA. If the application logic component is reverse-offloadable, it will be pulled back from the computing server to the target PC device; otherwise, the application logic component will stay on the computing server. It is a trade-off between paying a one-time transfer cost of migrating the application logic component (in the case of reverse-off loadable) vs. the continuous cost of communicating with that remote component in a Roamlet (in the case of nonreverse-offloadable).

\subsection{Transformation}

Transformation is a runtime UI generation process from a device-independent representation constructed by developers at design time to device-dependent presentations. Fig. 3(c) shows transformation of a deviceindependent UI component into a PDA presentation when the Roamlet migrates to a PDA. To build a device-independent representation and to generate devicedependent presentations, we provide a device-independent GUI library and a runtime transformation tool, which we called the SGUI toolkit.

The design of the SGUI toolkit is shown in Fig. 5. It contains three modules: SGUI library, transformation manager, and render manager. The process flow goes as follows. At design time, UI designers use widgets provided by the SGUI library to construct device independent UI presentations. At runtime, the
422

423

424

425

426

427

428

429

430

431

432

433

434

435

436

437

438

439

440

441

442

443

444

445

446

447

448

449

450

451

452

453

454

455

456

457

458 




Fig. 5. SGUI toolkit.

476 transformation manager transforms a device-indepen477 dent representation into a device-dependent presenta478 tion that satisfies the target device constraints (display 479 size and the available input methods). The render 480 manager displays the device- presentation on the target 481 device.

\section{2.3.1. Shop hunter application}

483 "Shop hunter" is a hypothetical multi-platform ap484 plication that helps a user to buy items anytime, any485 where. We will use the shop hunter application to 486 illustrate transformation adaptation supported by the 487 SGUI toolkit. The shop hunter application allows a user 488 to input new shopping items, to view a list of shopping 489 items, and to search for directions to nearby stores with the cheapest prices. We have prototyped a device-independent representation of the shop hunter UI using our SGUI toolkit, and applied transformation to generate device-specific presentations for notebook PCs, PDAs, and cell phones which are shown in Fig. 6.

The first noticeable difference between different device-specific presentations is how the device's displayable screen size changes the layout and pagination. The notebook PC presentation fits on one page, the PDA presentation is laid out on three different pages, and the cell phone presentation is broken up into six pages. The second noticeable difference between the screenshots is that the "sort" UI components are different between the notebook/Pocket PC presentation and the cell phone presentation. The notebook and Pocket PC presentations use a radio button group and the cell phone presentation uses a more compact drop-down list. The third noticeable difference is that the UI components corresponding to "add shopping item" is missing on the cell phone presentation, because this task involves text entries and is therefore inconvenient on a cell phone.

\subsubsection{SGUI library}

The SGUI library contains two modules: the virtual widget library and the SGUI events. The virtual widget
490

491

492

493

494

495

496

497

498

499

500

501

502

503

504

505

506

507

508

509

510

511

512

513



Fig. 6. Shop hunter application. 
514 library contains a comprehensive set of UI widgets 515 similar to Java's Swing library. It includes widgets such 516 as labels, buttons, menus, text fields, scroll bars, tables, 517 etc. The virtual widget library is implemented for each 518 Roam-supported device platform. Since the virtual 519 widget library is based on Swing, its APIs look very 520 similar to Swing APIs.

521 The SGUI events are abstractions of user interaction 522 events such as mouse, keypad, or stylus input. Since 523 different device platforms support different input meth524 ods, the SGUI library describes mappings between de525 vice-dependent events and abstract device-independent 526 events. This abstraction allows UI developers to handle 527 these events regardless of which platform a UI is run528 ning on. For example, an abstract action event associ529 ated with a button press can be generated from a mouse 530 click on a PC, a tap from stylus on a Pocket PC, or a 531 select-key press on a cell phone. The event is realized as 532 a device-dependent event at runtime, and then delivered to the application.

For some applications, mapping between device-dependent GUI events to device-independent events may not always be possible due to platform constraints. For example, an application may contain an interactive map that can zoom in or out when a user clicks on a specific point in the map image. On a PC, a mouse event can be used to capture the specific location of the mouse click. However, a cell phone lacks a pointing input method; as a result, it is not possible to map an equivalent event on a cell phone. To address the need for device-dependent events, the SGUI library also retains a set of events for device-dependent input methods. This allows a scalable application to enhance its UI on a particular device platform. Since device-dependent events are generated only on some specific device platforms, they should not be used to implement any core features of UI that are expected to be available on any devices.

\subsubsection{Device-independent representation}

UI designers specify two kinds of information in the device-independent representation-task model and layout. Fig. 7 depicts the top portion of the device-independent representation for the shop hunter application. The device-independent representation has a treelike structure. The child nodes of the root node are task nodes representing different tasks. For the shop hunter application, there are three tasks: add shopping item, view items, and search item. A task node can have subtask nodes, and so forth. The add shopping item task contains a scalable button called update list, and logical panel A. The button is a scalable UI component instantiated from the virtual widget library. A logical panel is a presentation unit that groups related child logical panels and scalable UI widgets. A spatial layout can be specified on the relative positions of widgets and panels contained in logical panels. For the add shopping item task node, UI designers would specify that logical panel $A$ be placed at the top, and update list button at the bottom. Logical panel A contains six smaller logical panels $A 1$ through $A 6$ arranged in a $2 \times 3$ grid.

UI designers can specify task preferences on task nodes. The task preference tells which platforms with which input methods are suitable to display this task. In the shop hunter application, a UI designer would specify the Add Shopping Item task to be suitable for displaying on notebook PCs or PDAs with virtual keyboards, but not for cell phones. As a result, UI components corresponding to Add Shopping Item task will not be displayed on cell phones as shown in Fig. 6.

In addition to layout, UI designers can specify other layout properties on logical panels, such as layout pri-



Fig. 7. Device-independent representation for the top portion of shop hunter application. 
584 ority, split-ability and title. Layout priority decides the 585 order in which logical panels will be placed on pages. 586 Split-ability decides if descendant nodes of a logical 587 panel can be placed on separate pages. By default, all 588 logical panels are not splittable. Titles of logical panels 589 are used to create a navigation menu bar for moving 590 between pages. For example, in the Pocket PC presen591 tation of the shop hunter application of Fig. 6, the 592 navigation menu bar Page Selection contains the titles of 593 logical panels as menus.

594 Integration of the layout structure and the task model 595 in the device-independent representation tree poses a 596 limitation that the task model hierarchy must not con597 flict with the layout structure hierarchy. It is not possible 598 to specify a task that places its widgets in the same 599 logical panel as widgets from another task. For example, 600 the view items task cannot place a widget under Logical 601 Panel A1 because it is also under Add Shopping Item 602 task. To address this limitation, we are looking into 603 separating the task model from the layout structure into 604 two presentation trees similar to Eisenstein et al. (2001). 605 Note that UI designers must construct device-inde606 pendent representations for both static and dynamic 607 content. In the following sections, we explain how the 608 transformation manager transforms a device-indepen609 dent representation into a device-specific presentation.

\section{2.3.4. Transformation manager}

611 The design of the transformation manager is shown 612 in Fig. 8. It contains three sub-modules: task manager, 613 layout algorithm and widget transformation. The first 614 step in transformation manager involves the task man615 ager. Its job is to choose the appropriate tasks for the 616 target device platform, and to remove widgets belonging 617 to tasks not appropriate for the target device platform. 618 For example, if UI designers specify that the Add 619 Shopping Item task in the shop hunter application is not 620 suitable for cell phones; widgets corresponding to the 621 Add Shopping Item task are removed by the task man622 ager and not displayed on the cell phone presentation.

623 The second step in the transformation manager in624 volves the layout algorithm working with widget trans625 formation. Its job is to paginate the presentation into 626 separate pages according to the layout specified by UI 627 designers and also to meet the constraint that a page 628 cannot exceed the target device's displayable screen size.

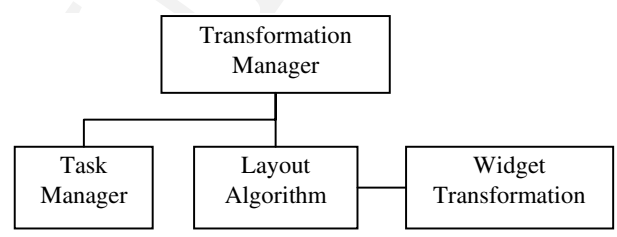

Fig. 8. Transformation manager design.

\subsubsection{Layout algorithm}

629

We define two requirements for our layout algorithm. First, the generated presentation should have reasonably high quality without requiring significant device-specific customization from UI developers. One implication of high quality and the display size constraint is that the generated presentation must fit the target display without scrolling, because scrolling generally degrades usability. Second, the algorithm must be fast, so that it does not cause significant presentation generation delay. There have been many methods on how to layout widgets for different display sizes. Some of these methods, such as the one proposed by Masui (1994), are highly computationally complex. Some methods require too much information from developers: for example, $\mathrm{Hu}-$ manoid (Szekely et al., 1992) asks developers many layout-related questions before it can generate a final presentation. Among all proposed methods, $\mathrm{T}_{\mathrm{E}} \mathrm{X}$ (Linton, 1989) is the most promising method in formatting two dimensional box-like GUI widgets (Masui, 1994; Olsen et al., 2000). $\mathrm{T}_{\mathrm{E}} \mathrm{X}$ allows each widget to report its desired size for positioning.

Fortunately, Java has default layout managers that are similar to $\mathrm{T}_{\mathrm{E}} \mathrm{X}$. Java also allows UI developers to specify the desired location of each widget through a set of predefined layout constraints. In order to meet our first requirement, we propose to have only one set of layout specification from UI developer, and this set of layout specification is used to generate layouts for other platforms. The specification is the same as the Grid Bag Layout Constraint in Java. We recommend UI developers to specify the layout according to the presentation on the largest device display, such as a presentation on a $\mathrm{PC}$, for reasons detailed in Section 3.6. The layout algorithm will try to follow that specification as closely as possible when it is creating layouts for other platforms with smaller display sizes.

The general strategy for the layout algorithm is to start from the lowest nodes in the device-independent representation tree, and then work its way up to the root node. When it encounters a non-splittable logical panel that cannot fit the display size using the specified grid bag layout, the layout algorithm applies flow layout. We choose flow layout because it is simple, and it involves minimum computation while maintaining a reasonable presentation (Vanderdonckt, 1995). Since using flow layout may not generate a high quality layout, it is only applied when the specified grid bag layout fails. If flow layout also fails on a non-splittable logical panel, the layout algorithm may apply widget transformation to find smaller alternative presentations. The detailed steps of the layout algorithm are described as follows:

1. The algorithm starts by finding the lowest-level unsplittable node with the highest layout priority in the 682 presentation tree as shown in Fig. 9. A lowest-level 683 


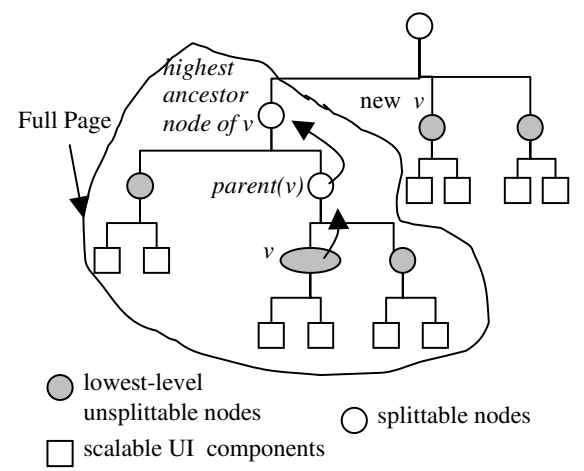

Fig. 9. Layout algorithm.

684

685

712 4. If the size of the page is smaller than the size of the

unsplittable node is defined as a node whose child nodes are all widgets (or leaf nodes), and the widgets have to be placed on the same page. The goal is to try to place widgets that are assigned higher layout priorities to the first few pages, so that they are easier for users to locate. Denote $v$ as the chosen node.

2. A default style corresponding to the target device platform is applied to the set of widgets under node $v$. The style guide sets common properties of widgets such as font size, spacing between components, etc. The purpose of the style is to have a consistent look across different widgets. The set of widgets under node $v$ are laid out on a page according to the grid bag layout constraints specified by the UI developer. The precise size of the page is then calculated.

3. If the size of page is bigger than the device display size, the page is over-filled. We apply flow layout on child nodes under $v$, and re-compute the page size. If the page is still over-filled, we check if the node is splittable or not. If the node is splittable, we allocate a new page to place extra widgets that cannot fit on the previous page. If the node is non-splittable, widget transformation is applied to as many child nodes under $v$ as needed such that the flow layout can fit them on one page. If widget transformation also fails, the layout algorithm will leave some widgets out of the page and notify its users. Details on widget transformation are discussed in Section 2.3.7. device screen size, the page is under-filled. This means that the algorithm can accommodate additional widgets from sibling nodes of node $v$. The algorithm proceeds to find the highest ancestor node of node $v$ in the presentation tree as shown in Fig. 9, such that using that ancestor node can pack the maximum number of widgets in a page without violating the display size. Denote the parent node of node $v$ as PARENT(v). The algorithm tries to place the set of widgets under $\operatorname{PARENT}(v)$ on a page. This is done by repeating step 2 , i.e., applying the current style and the grid bag layout specification to the widgets under PARENT( $v$ ). If the resulting page is still under-filled, it tries $P A R-725$ $\operatorname{ENT}(P A R E N T(v))$, and so forth until the resulting 726 page becomes full.

5. If the page is full, a new page is allocated for placing remaining widgets. The algorithm first finds another lowest-level unsplittable node with the next highest task priority from the presentation tree that hasn't been placed on any pages. This node becomes the new node $v$, and the algorithm goes to step 2 .

6. When all nodes are processed-that is, placed on a page - the algorithm terminates.

For the shop hunter application in Fig. 6, the table showing the shopping list has different device-specific presentations on Pocket PCs and cell phones. For the cell phone presentation, the table is transformed into a selection list using item names as indices. The map image is scaled down on the Pocket PC presentation and on the cell phone presentation. Techniques involving scaling images and graphic objects (W3C, 2000) are outside the scope of this paper.

\subsubsection{Page navigation}

While the layout algorithm is automatically generating a new layout, it is also constructing a simple navigation menu bar for moving between pages. If the application already has a menu bar, the algorithm will simply add a customizable menu named "Page Selection" by default as the first menu from the left on the menu bar. If the application does not have any menu bar, a new one is created. On the Pocket PC, the menu bar always appears at the top of each page under the Page Selection menu bar as shown in Fig. 6. On the cell phone, the first page is always the top-level task navigation menu. For each generated page, a menu item is created and it will be automatically added as the item of the "Page Selection Menu." The name for each menu item is from the title property of the occupying logical panel.

\subsubsection{Widget transformation}

Widget transformation is the transformation from one widget, either primitive or composite, to another that consumes less space in the graphical layout. Transformation is guided by transformation rules, similar to rules in a rule-based system. Widget transformation rules are selectively applied to yield a presentation which is optimally usable given display size constraints. For example, Fig. 10 shows the effect of a transformation rule mapping a large, composite widget containing pairs of property labels and value textfields, into a smaller composite widget containing a property list box and a single value textfield. The transformed composite widget is smaller and remains easy to use. 


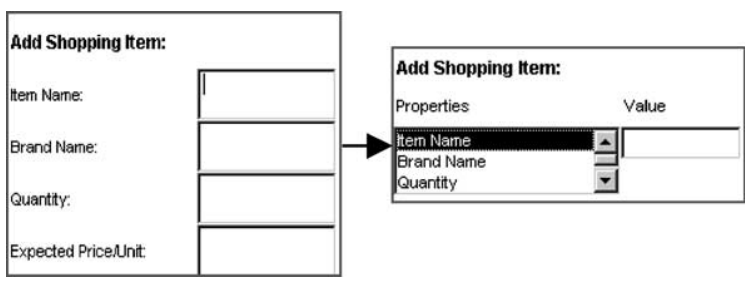

Fig. 10. A multiple-to-multiple transformation.

At the core of widget transformation is an algorithm to determine which widgets to transform, and which transformation rules to apply to them.

To determine the widgets that are more acceptable to transform, developers specify which widgets are corerepresenting important application functionality, and which are optional-less important for use of the application. These hints are provided for primitive widgets, as well as those that are combined into composite widgets. We prefer to transform optional widgets rather than core widgets whenever possible, so that we degrade usability as little as possible (Vanderdonckt, 1995); only when there are no optional widgets, or when the layout size reduction is insufficient to meet display size constraints, are core widgets transformed.

Determining the transformation rules to apply to the widgets is more complex. There are four types of transformation rule:

- One-to-one: transforms from a single primitive widget to another single widget, e.g., a rule transforming a list into a drop-down box.

- One-to-multiple: transforms from a single widget into multiple widgets, e.g., a rule transforming a single table into multiple lists or drop-down boxes.

- Multiple-to-one: transforms from multiple widgets of the same type into a single widget, e.g., a rule transforming a group of radio buttons or checkboxes into a single drop-down list box, and a rule transforming a group of one-line textfields into a single multi-line textarea.

- Multiple-to-multiple: transforms from multiple widgets of different types into other multiple widgets, e.g., a rule transforming a group of label and textfield pairs into a drop-down list box and a single textfield, as shown in Fig. 10. Note that a multiple-to-multiple rule is composed of a set of multiple-to-one rules, and a set of classic relationships described in Vanderdonckt (1995).

Each of these classes of transformation rule has a priority assigned to it, corresponding to the demonstrated usability of widgets transformed by rules in the class. That is, we prefer transformation rules that tend to result in easier-to-use widgets over transformation rules that tend to result in harder-to-use widgets. One- to-one rules are preferred first, then one-to-multiple, multiple-to-one, and finally multiple-to-multiple. Note that multiple-to-multiple rules can change the UI drastically, and degrade usability, compared to one-to-one rules. Conversely, note that one-to-one rules tend to offer less space savings than multiple-to-multiple rules. Our prioritization balances this tradeoff: we want as large - and consequently as usable - a UI as possible given display size constraints, but we accept smaller and less usable UIs as necessary.

We limit the set of transformation rules that may be applied by eliminating rules whose original widgets require input methods that are unsupported by the transformed widgets on the target device. For example, if the original UI contains a J2ME DoJa button that has an action triggered on a mouse-in event, any rules transforming that button to a softkey are not applicable, because softkeys do not support any equivalent to mouse-in events. The current SGUI toolkit provides a set of commonly used transformation rules. We also allow developers to add their own transformation rules.

\subsubsection{SGUI state and event transformation}

When an application is migrated from a device of one platform to another device of a different platform, the presentation on the target device may use a different set of widgets from the source device, so there is a need to map running state between the source presentation and the target presentation. This is done by state synchronization and restoration between the running state of a device-specific widget and its corresponding device-independent widget. These two mechanisms are supported in the SGUI library to ensure that the device-independent representation always has the most recent widget state right before and right after any migration. Prior to any application migration, the running state of a devicespecific widget is synchronized with its corresponding device-independent widget. After an application migration, the running state of the device-independent widget is restored to the device-specific widget.

Event transformation is also needed to support the same UI interaction across different device-dependent presentations. This is done by a bi-directional mapping mechanism between device-dependent events generated from device-dependent widgets and their corresponding device-independent events generated by a device-independent widget. This mapping mechanism is supported in our SGUI library.

\subsection{Security}

We assume that Roam agents have been installed securely and they are trusted entities. We also assume that a user would only migrate applications among his/ her personal devices (not public and not shared) such as cell phones, PDAs, and home PCs. This means that a 


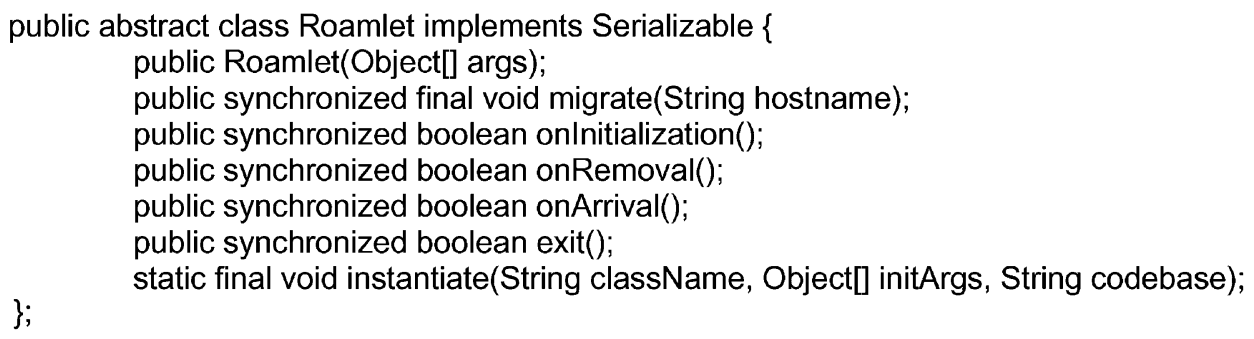

Fig. 11. Roamlet runtime migration API.

user should know when to expect an application migration on which device and from which device.

Based on these assumptions, we have applied an authenticated encryption scheme (Rogaway et al., 2001) that can provide both privacy and authentication. Privacy means that application execution state is encrypted so that only the intended recipient with the correct password can decrypt it. Authentication means that the intended recipient can distinguish a legitimate application migration sent by its user from a malicious application migration sent by other users. Roam's security works as follows. At the start of the application migration, the Roam agent running on the source device prompts the user for a password. This password is a one-time password that is valid only for one application migration. At the same time, it will also generate a onetime number called a nonce. The only requirement for the nonce is that it is a new number for each application migration. The nonce can be generated from a random number generator or a simple counter that increments each time it is used. Based on the one-time password and nonce, the application execution state is encrypted as ciphertext. The ciphertext is sent together with the cleartext nonce to the target device. On the target device, the Roam agent asks the user for the same password that was used to encrypt the ciphertext on the source device. Based on the password and the nonce, the Roam agent can decrypt the ciphertext to get the application execution state. If the password used in decryption does not match the password used in encryption, the authenticated encryption algorithm returns "INVALID" to the Roam agent and the migration request is denied.

906 We have implemented the Roam system using the 907 Java language and the Java language features: RMI, 908 Serialization, and Reflection. The Roam system cur909 rently runs on the PCs with JVM, and on Pocket PC 910 (PDA) devices with Personal Java VM. In the future, we 911 are planning to port the Roam system to other JVMs 912 with RMI and Serialization. The total code size of the 913 Roam system is approximately 15,000 lines, with 8500 914 lines in the SGUI toolkit.
The Roam system provides a set of Roamlet APIs. The core class in the Roamlet APIs is called Roamlet, which all Roamlets must extend. The Roamlet APIs allow a Roamlet to migrate at runtime, to be instantiated dynamically, to offload computation, or to be transformed. We describe the essential Roam APIs here.

\subsection{Roamlet runtime migration API}

The runtime migration API is encapsulated in a class called Roamlet shown in Fig. 11. All Roamlets must extend the class Roamlet. A Roamlet calls the migrate( ) method to be dispatched to a target device specified by the hostname parameter. The Roam agent calls the Roamlet's onlnitialization( ) method when it is first instantiated on a device. The Roam agent calls the Roamlet's onRemoval() method before it is removed from the source device, and after it migrates successfully to a target device. The Roam agent calls the Roamlet's onArrival( ) method when it arrives at the target device. A Roamlet calls the exit( ) method to remove itself from the Roam system. The instantiate( ) method is called to instantiate a Roamlet on a local host. After a Roamlet is instantiated on a device, the Roam agent creates a separate thread to run it.

\subsection{Roamlet component description API}

A Roamlet can describe its components using the API shown in Fig. 12. A Roamlet calls addComponentDesc() to add a component descriptor, RoamletComponentDesc, for each of its components. RoamletComponentDesc describes the type of component (type, which can be M-DD, S-DD, or S-DI), the class name of this component, the class name of the component to be instantiated (classname), and a list of possible implementations for this component. Each implementation of a component is described in a separate ImplDesc, which contains the class name (classname) of the implementation and the device requirement for this implementation (req).
915

916

917

918

919

920

921

922

923

924

925

926

927

928

929

930

931

932

933

934

935

936

937

938

939

940

941

942

943

944

945

946

947

948

949

950

951 




Fig. 12. Roamlet component description API.

\section{3.3. SGUI API}

953 The SGUI library provides four packages (sgui, 954 sgui.event, sgui.dd.event, and sgui.ir) for UI developers. 955 The sgui package provides virtual widgets similar to 956 widgets available in the Swing library with prefix S, such 957 as SButton, SCheckbox, STextfield, SLabel, etc. The 958 sgui.event package provides device-independent events 959 with S prefix, such as SActionEvent. The sgui.dd.event 960 package provides device-specific events, such as 961 SMouseEvent. UI developers follow the Java 2 event 962 model to add SGUI event listeners and specify actions 963 upon the reception of SGUI events.

964 The sgui.ir package provides APIs for constructing a 965 device-independent representation. One of the core 966 classes is the LogicalPanelNode shown in Fig. 13. It has 967 an add( ) method to add virtual widget and logical 968 panels as its child nodes. A LogicalPanelNode can 969 specify a list of properties, such as layout constraint, 970 task preference, layout priority, whether it can be pag971 inated across multiple pages, and whether it represents 972 core or optional features.

\section{3.4. Chess Roamlet}

974 We have taken a Java-based Chess game that is freely 975 available on the Internet and rewritten it as a Roamlet. 976 The Chess game has two modes: multiplayer mode 977 where two users play against each other, and computer 978 mode where a user plays against AI. The mobile envi979 ronment is setup as in Fig. 14: a Pocket PC device running Personal Java VM, a notebook PC running a 980 standard J2SE VM, and they are both connected by an 981 802.11 wireless LAN. The user starts the Chess game in 982 computer mode on the notebook PC and then migrates 983 it to the Pocket PC. There is a third device, a desktop 984 $\mathrm{PC}$, acting as the remote server where the AI component 985 can be offloaded to.

The Chess Roamlet contains the following components:

- An application logic component is implemented as an offloadable device-dependent component (S-DD). It 990 runs an AI engine that calculates the computer's next 991 move by building a search tree, which is both compu- 992 tationally and memory-intensive. It has a device re- 993 quirement of a PC.

- A GUI component is implemented as a non-offloadable device-independent component (S-DI) using the SGUI library. It draws a player board that holds both a chessboard and chat/message boxes where two players can send messages to each other.

- A second GUI component is implemented as a nonoffloadable device-dependent component with two implementations (M-DD). These two implementations have two different sets of images showing chess pieces, the set for the PC presentation has larger images than that of the Pocket PC presentation.

The Chess game in computer mode with PC presentation is shown on the left side of Fig. 15. When the user migrates the Chess game to the Pocket PC, the first (S881 82 883 984 985



Fig. 13. Sample APIs for constructing device-independent representation. 




Fig. 14. Experimental setup of Chess Roamlet.

1009 DI) GUI component is transformed into the Pocket PC 1010 presentation shown on the right side of Fig. 15. Since the 1011 player board is too large for the Pocket PC's display, 1012 SGUI layout algorithm paginates it into two pages. The 1013 first page shows the chessboard, and the second page 1014 shows chat and message boxes. A page navigation menu 1015 is added to the right of the menu bar for users to switch 1016 between two pages. The M-DD GUI component is in1017 stantiated with the Pocket PC presentation and it loads 1018 the smaller set of images for chess pieces. The applica1019 tion logic component is offloaded to the computing 1020 server.

\section{3.5. Experience on development effort and performance}

1022 The Chess game was originally implemented to run 1023 on the PC using Java AWT GUI library. We have asked 1024 third party developers, who are new to Roamlet pro1025 gramming, to rewrite the Chess game as a Roamlet us1026 ing SGUI library. The experience from the third party 1027 developers have told us that most of the efforts in re1028 writing the Chess application fall into the following 1029 three categories: (1) modularizing an application into 1030 application logic components and GUI components, and converting them into Roamlet components, (2) porting GUI components to use the SGUI library, and (3) adding multiple device-specific GUI components for platforms that are not supported by the original Chess application. Their experience also told us that it is relatively straightforward to separate application logic components from UI components in a well-written Java application. The difficult part is to decide the type (S-DI, S-DD, or M-DD) for each component. Finally, their experience told us that, since our SGUI library follows Java Swing library, porting AWT components to use SGUI components is relatively straightforward.

The original Chess game has approximately 7900 lines of code. After the converting it into a Roamlet, it has approximately 9000 lines of code, which is about $14 \%$ increase in code size. Most of the additional code comes from code that converts Java objects into Roamlet components, and the second (M-DD) GUI component that supports the Pocket PC presentation. This shows the tradeoff between development cost and UI quality. That is device-dependent UIs in general have higher quality, but developers need to create M-DD presentations for different platforms, resulting in increased application code size. This tradeoff can also be found in the Connect4 game. When its UI component has two device-dependent implementations for PC and PDA, the code size is approximately 1200 lines. The code size is reduced to 1100 lines when its UI component is re-implemented as a device-independent component using SGUI library.

We have measured migration latency of application migration. Migration latency is defined as the elapsed time from the moment the user accepts the migration request on the target device, to the moment when the Roamlet is completely restored on the target device. We have measured latency for both Connect 4 and Chess games migrating from a notebook PC (Intel Pentium III-800 CPU running Windows XP) to a Compaq iPAQ H3870 Pocket PC (StrongARM 206 MHZ CPU running
1031

1032

1033

1034

1035

1036

1037

1038

1039

1040

1041

1042

1043

1044

1045

1046

1047

1048

1049

1050

1051

1052

1053

1054

1055

1056

1057

1058

1059

1060

1061

1062

1063

1064

1065

1066

1067

1068

1069
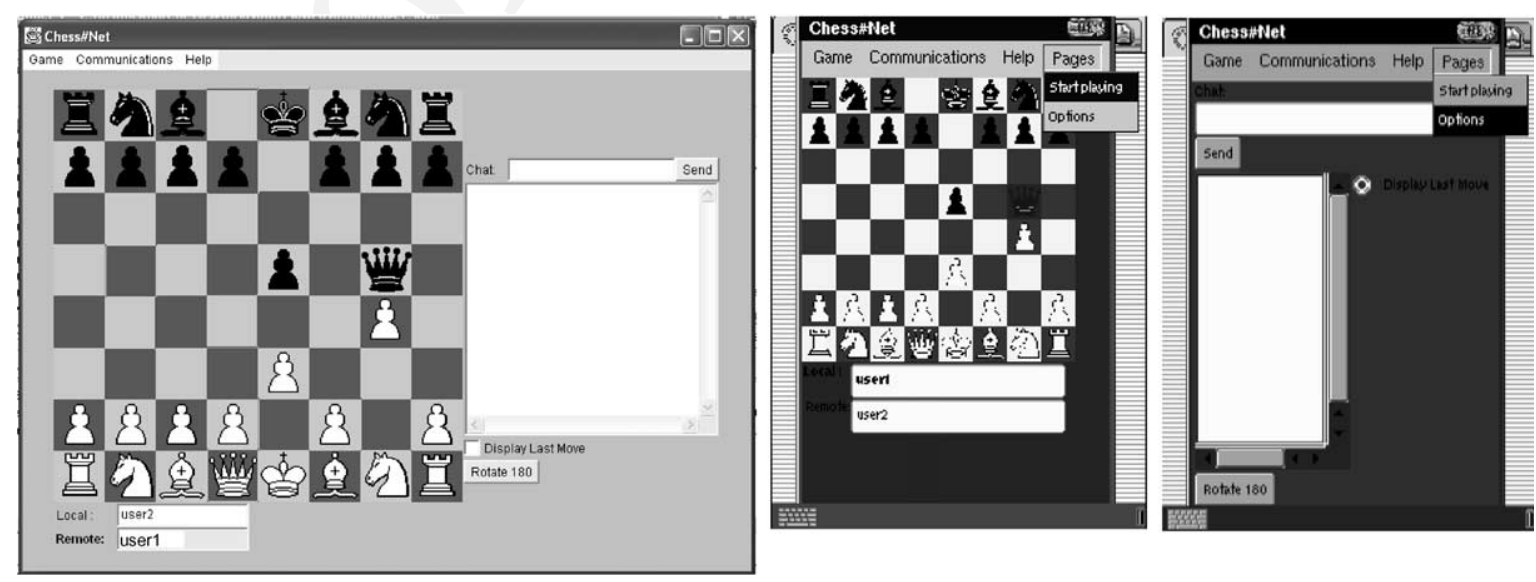

Fig. 15. Screenshots of Chess game on notebook PC and Pocket PC after migration. 
Table 3

Migration latency measurements

\begin{tabular}{|c|c|c|c|c|}
\hline \multirow[t]{3}{*}{ Applications } & \multicolumn{4}{|l|}{ Migration latency } \\
\hline & \multicolumn{2}{|l|}{ Notebook PC to Pocket PC } & \multicolumn{2}{|l|}{ Pocket PC to notebook PC } \\
\hline & Context transfer time (s) & Transformation time (s) & Context transfer time (s) & Transformation time (s) \\
\hline Chess & 4.9 & 10.2 & 4.2 & 1.1 \\
\hline Connect4 & 2.8 & 6.4 & 1.2 & 1.4 \\
\hline Shop hunter & N/A & 3.7 & N/A & 0.9 \\
\hline
\end{tabular}

1070 Linux) and vice versa. We divide the migration latency

1071 into two parts: (1) context transfer time is the time for

1072 saving the execution state on the source device, sending

1073 and restoring it on the target device, and (2) GUI

1074 transformation time is the time for transforming a de-

1075 vice-independent GUI component into a target device-

1076 specific presentation. The measurements are shown in

1077 Table 3.

1078 Measurements show that transformation time domi-

1079 nates the migration latency when migration is from a

1080 notebook PC to a Pocket PC, because the layout algo-

1081 rithm is computationally intensive and it runs the target

1082 device which is a slower Pocket PC. However, when

1083 migration is from a Pocket PC to a notebook PC, the

1084 context transfer time dominates the migration latency.

1085 This is due to serialization of the Roamlet execution

1086 time on a slow Pocket PC. For the shop hunter appli-

1087 cation, transformation is offloaded to a PC. Measure-

1088 ments show that the transformation manager takes less

1089 time to generate a presentation for a bigger display (0.9

$1090 \mathrm{~s})$ than for a smaller display (3.7 s). The reason is that

1091 generating a presentation for a smaller display is likely

1092 to involve testing different paginations and transfor-

1093 mation rules.

\subsection{End-user usability}

Our limited experience on the available Roamlet applications shows that SGUI toolkit can generate consistent presentations across different platforms. There are three aspects of consistencies: task consistency, layout consistency, and transformation consistency. Task consistency means that, unless the developers specify that some tasks are not appropriate on certain platforms, all tasks will be presented across all platforms. Layout consistency means that, if UI components are laid out next to each other on one platform, they are likely to be found adjacent to each other or on adjacent pages on other platforms. Transformation consistency means that users can expect similar transformations to be applied to same type of UI components across applications. We believe that these consistencies contribute to better learnability of applications.

Our simple page navigation menu works only for simple UIs but not complex UIs. We believe that we can improve the usability and efficiency of generated page navigation by incorporating device-specific navigation models provided by SGUI toolkit or explicitly specified by UI developers. To generate better page navigation, the navigation model should consider interactions and relation between tasks and device's input methods.

\section{Related work}

We divide the related work into two parts: mobile agent systems and multi-platform UIs.

\subsection{Mobile agent systems}

There has been an abundance of research work in the area of migrateable mobile agent systems. Aglet (Lange and Oshima, 1998) is one of the most well-known Javabased agent systems. It provides a Java development toolkit and libraries for building mobile agents that can move from one computer to another. Like Roamlet, Aglets do not require any modifications to the Java VM. An Aglet is shielded through an Aglet Proxy, which protects the Aglet from unauthorized access. Aglets run in an execution environment called AgletContext, which is like the Roam agent. However, Roamlets differ from Aglets in that Roamlets assume a heterogeneous device environment, whereas the Aglet assumes a homogeneous PC or PC-equivalent environment. Furthermore, the Roam system implements dynamic instantiation and computation apportioning to address the device heterogeneity problem. We have found similar Java-based agent systems in Jumping Beans (Aramira, 1999), MOA (Milojicicc et al., 1998), Concordia (Mitsubishi Electric ITA Horizon Systems Laboratory, 1998), Voyager (ObjectSpace, xxxx), Mole (Strasser et al., 1996) and Telescript (White et al., 1997). Voyager is a commercial product by ObjectSpace, and it incorporates features of mobile agents and mobile objects into the ORB and COBRA. MOA performs resource management on migrateable applications as to what system resource they can use on the target device, and it also supports migrateable communication channels (the external running state) so that running communication channels can migrate with the rest of the applications. There are also similar but non-Java-based agent systems, e.g., AgentTcl (Gray et al., 1997).
1114

1115

1116

1117

1118

1123

1124

1125

1126

1127

1128

1129

1130

1131

1132

1133

1134

1135

1136

1137

1138

1139

1140

1141

1142

1143

1144

1145

1146

1147

1148

1149

1150

1151

1152

1153

1154 
1155 Fünfrocken (Fünfrocken, 1998) and Ara (Peine and 1156 Stolpmann, 1997) describe ways to achieve strong mi1157 gration for Java applications. Strong migration means 1158 that the call stack, which is a part of the thread state that 1159 is not serializable, can also be migrated. Fünfrocken 1160 uses a preprocessor that adds code to capture and to 1161 restore the state of the program stack at various exe1162 cution points. Ara proposes modifications to the JVM 1163 interpreters. Since thread state migration is not a specific 1164 challenge for heterogeneous device, the Roam system 1165 supports only weak mobility.

1166 Sumatra (Acharya et al., 1997) is an extension of Java 1167 that supports resource-aware migrateable mobile appli1168 cations. Sumatra's resource-awareness is based on a 1169 monitor-feedback-adaptation loop. A resource monitor 1170 watches the level and quality of the system resources, 1171 and provides updates to the applications either contin1172 uously or on-demand. Applications can then adapt 1173 based on the resource updates, e.g., migrating some 1174 threads to remote devices to perform the computation. 1175 Sumatra is focused on building adaptive applications in 1176 the PC or PC-equivalent device environment, and ad1177 aptation is based on runtime resource level and quality. 1178 This is different from the Roam system, which is focused 1179 on adaptation in a heterogeneous device environment. 1180 Roamlets adapt according to target device capability at 1181 migration time and load time only.

1182 We have found parallel and ongoing research efforts 1183 at IBM (Banavar et al., 2000). The IBM researchers 1184 have proposed a new application model for pervasive 1185 computing. They have described several research chal1186 lenges, e.g., device-specific rendering and application 1187 apportioning, which are similar to the problems that the 1188 Roam system is addressing.

\section{4.2. Multi-platform user interfaces}

1190 The model-based approach offers an attractive alter1191 native to build a high-level tool for multi-platform UIs 1192 (Szekely, 1996). In model-base systems such as $\mathrm{Hu}-$ 1193 manoid (Szekely et al., 1993), ITS (Wiecha et al., 1990), 1194 UIDE (Sukaviriya et al., 1993), and Mickey (Olsen, 1195 1989), UI designers would use a declarative language to 1196 specify abstract and high-level models that describe what 1197 UI should be. The model-base system would automati1198 cally generate low-level UI executable code. The model1199 based technique can be exploited for multi-platform UI 1200 generation. For example, UI designers can specify the 1201 high-level models using AIOs (abstract interactive ob1202 jects), which are device-independent objects. The AIOs 1203 are mapped to device-dependent CIOs (concrete inter1204 active objects) supported by the target platform UI li1205 brary. This AIO-CIO selection technique has been 1206 illustrated in model-based systems for purposes other 1207 than multi-platform UI generation. For example, Hu1208 manoid uses a "replacement hierarchy" for selecting the most appropriate presentation template for displaying a semantic object. UIDE contains constraints on how to select the most appropriate interface actions to represent application actions specified by the UI designers in application models.

In recent work, RedWhale software (Eisenstein et al., 2000, 2001) employs the model-based approach by introducing abstract models that specifically target multiplatform UIs. It is comprised of platform model, presentation model, and task model. The platform model allows the UI designers to specify platform constraints that the UI generation must follow, such as device screen sizes, input methods, etc. The presentation model specifies the visual appearance of the generated UI, which may include the layout of the UI components and the mapping between AIOs and CIOs. The task model specifies the decomposition of big tasks into smaller subtasks. The device-dependent UI generation is based on all three models.

Another recent model-based work is Paternò et al's ConcurTaskTree (Paternò, 2000). Its task model contains user tasks, abstract tasks, interaction tasks, and application tasks. Each task is related to each other via a set of temporal relationships such as enabling, deactivation, and iteration. The ConcurTaskTree is generally accepted to be a powerful task model to construct UIs. However, it is mainly targeted to single platform UIs. If we try to apply ConcurTaskTree to generate multiplatform UIs, all UIs will have the same interaction pattern as the interaction tasks are tightly coupled in the model. However, devices such as cell phones and PDAs have distinctive interaction patterns. To solve this problem, our device-independent representation only has user tasks. The interaction pattern information is expressed as properties of the task, e.g., task preference, or as platform-specific interaction models that would interact with the task model. The latter expression will be our future work. With our task model, we can easily customize the interaction pattern for each platform.

Although the model-based approach is an attractive technique for creating SGUI tools, it has usability and authoring issues (Myers, 1995) such as loss of finegrained control of UI details, the quality of generated UI, and the time needed for UI designers to learn and construct models. Our SGUI toolkit combines the model-based approach with the benefits of traditional widget-based UI programming, as a solution to some of these issues. Programming with the SGUI library is very similar to programming with a traditional widget library such as Swing, because the SGUI library is consisted of low-level UI widgets rather than abstract models. The transformation in SGUI is at the level of widgets and events, rather than from abstract interaction models to widgets and events. This has additional benefits that no code generation is necessary, and that complex models 
1264 of UI Logic (data flow constraints, sequencing, side ef1265 fects, etc.) (Szekely et al., 1992) are also not needed.

1266 Microsoft has a commercial product available called 1267 the. NET Mobile Internet Toolkit (Microsoft Corpo1268 ration, xxxx), which targets different mobile devices 1269 ranging from the more capable PocketPC platform to 1270 relatively simple pagers. The Mobile Toolkit does not 1271 use a model-based approach. Rather, it assumes devel1272 opers have already implemented desktop PC versions of 1273 the user interface components, and that they want to 1274 port from existing code. The Mobile Toolkit allows 1275 developers to specify the corresponding UI presentation 1276 on mobile platforms, but the layout specification is very 1277 limited. It only offers flow-based layout (rather than the 1278 more sophisticated Grid-Bag layout). In addition, de1279 velopers must group widgets together into a form, the 1280 function of which is very similar to our LogicalPanel1281 Node. However, .NET mobile forms cannot be placed 1282 on the same page even if a page has enough space to 1283 accommodate them.

\section{5. Conclusion}

1285 In this paper, we present challenges, design, and im1286 plementation of the Roam system. The Roam system is 1287 a seamless application framework for building seamless 1288 applications that can migrate at runtime across hetero1289 geneous devices. The Roam system provides adaptation 1290 strategies at the component level, including dynamic 1291 instantiation, offloading computation, and transforma1292 tion.

1293 There are many future directions to improve the 1294 Roam system. One problem with the existing SGUI 1295 toolkit is that it is difficult to customize a device-inde1296 pendent representation for a particular device. The 1297 reason is that the device-specific customization requires 1298 developers to add transformation rules that are both 1299 device-specific and application-specific. When the de1300 velopers change the device-independent model at a later 1301 time, they may also have to update these transformation 1302 rules that are affected by the change.

1303 We also like to support seamless application migra1304 tion for real-time applications such as video conferenc1305 ing. For example, a use may want to migrate a video 1306 conferencing from a mobile phone to a car navigation 1307 system when he/she is entering a car. This places a real1308 time constraint on migration latency. We want to make 1309 sure that the interruption time is minimized during ap1310 plication migration. One possible approach is to hide the 1311 migration latency, by delaying the application termina1312 tion on the source device, until adaptation has been 1313 applied to the application components on the target 1314 device.

1315 Finally, we would like to support a Roam execution 1316 state repository service where a user can save a Roam application on one device, and restore it at a later time on any device. This overcomes the limitation in the current Roam system where application migration cannot be suspended. We would like to extend this to collaborative applications where each user can save and restore application execute state individually without affecting other users.

\section{Uncited references}

Chu et al. (2001), NTT DoCoMo (2001), Sukaviriya et al. (1994), Sun Microsystems (1998, 2000a,b) and Wiebus (xxxx).

\section{Acknowledgements}

We would like to thank Dan Rosen for his help in proof-reading this document. We would also like to thank reviewers of JSS for their comments.

\section{References}

Acharya, A., Ranganathan, M, Saltz, J., 1997. Sumatra: a language for resource-aware mobile programs. Mobile Object Systems, Lecture Notes in Computer Science April, 1997.

Aramira, Inc., 1999. Jumping Beans ${ }^{\mathrm{TM}}$ White Paper, October 1999. Available from <http://www.jumpingbeans.com/index.html>.

Banavar, G., Beck, J., Gluzberg, E., Munson, J., Susman, J., Zukowski, D., 2000. Challenges: an application model for pervasive computing. In: Proceedings of the 6th Annual International Conference on Mobile Computing and Networking, Boston, MA, August 2000, pp. 266-274.

Chu, H., Song, H., Wong, C., Kurakake, S., 2001. Seamless Applications over Roam System, UbiTools'01 (Part of ACM UbiComp'01), September 2001. Available from <http:// choices.cs.uiuc.edu/UbiTools01/>.

Eisenstein, J., Vanderdonckt, J., Purerta, A., 2000. Adapting to mobile contexts with user-interface modeling. In: Proceedings WMCSA'00, December 2000.

Eisentein, J., Vanderdonckt, J., Puerta, A., 2001. Applying modelbased techniques to the development of uis for mobile computers. In: Proceedings IUI' 01, January 2001.

Fünfrocken, S., 1998. Transparent migration of Java-based mobile agents (capturing and reestablishing the state of Java programs). In: Proceedings of the 2nd International Workshop on Mobile Agents, Stuttgart, Germany, September, 1998.

Gray, R.S., Kotz, D., Nog, S., Rus, D., Cybenko, G., 1997. Mobile agents for mobile computing. In: Proceedings of the Second Aizu International Symposium on Parallel Algorithms/Architectures Synthesis, Fukushima, Japan, March, 1997.

Lange, D.B., Oshima, M., 1998. Mobile agents with Java: the Aglet API. World Wide Web Journal.

Linton, A., Vlissides, M., Calder, R., 1989. Composing user interfaces with interviews. IEEE Computer 22 (2).

Masui, T., 1994. Evolutionary learning of graph layout constraints from examples. In: Proc. of ACM UIST'94, November 1994, pp.103-108.
1333

1334

1335

1336

1337

1338

1339

1340

1341

1342

1343

1344

1345

1346

1347

1348

1349

1350

1351

1352

1353

1354

1355

1356

1357

1358

1359

1360

1361

1362

1363

1364

1365

1366

1367 
Microsoft Corporation, Best Practices for the Microsoft Mobile Internet Toolkit Image Control. Available from <http://msdn.microsoft.com/theshow/Episode023/default.asp>.

Milojicicc, D.S., LaForge, W., Chauhan, D., 1998. Mobile objects and agents (MOA). In: Proceedings of the 4th USENIX Conference on Object-Oriented Technologies and Systems (COOTS). Santa Fe, New Mexico, April, 1998.

Mitsubishi Electric ITA Horizon Systems Laboratory, 1998. Mobile Agent Computing A White Paper, January 1998.

Myers, B., 1995. User interface software tools. ACM Transactions on Computer Human Interaction 2 (1), 64-103.

NTT DoCoMo, Inc., 2001. i-mode Java Content Developer's Guide, May 2001.

ObjectSpace, Inc., Voyager. Available from <http://www.objectspace.com/products/voyager>.

Olsen, D., 1989. A programming language basis for user interface management. In: Proceedings of CHI'89, Austin, Texas, May 1989, pp. 171-176.

Olsen, D., Jefferies, S., Nielsen, T., Moyes, W., Fredrickson, P., 2000. Cross-modal Interaction using XWeb. In: Proc. of ACM UIST'00, November 2000.

Paternò, F., 2000. Model-Based Design and Evaluation of Interactive Applications. Springer-Verlag, London.

Peine, H., Stolpmann, T., 1997. The architecture of the ara platform for mobile agents. In: Proceedings of the 1st International Workshop on Mobile Agents, Berlin, Germany, April 1997.

Rogaway, P., Bellare, M., Black, J., Krovetz, T., 2001. In: Eighth ACM Conference on Computer and Communications Security (CCS-8). ACM Press, pp. 196-205.

Strasser, M., Baumann, J., Hohl, F., 1996. Mole-a Java based mobile agent system. In: 2nd ECOOP Workshop on Mobile Object Systems, Linz, Austria, July 1996, pp. 28-35.

Sukaviriya, P., Foley, J.D., Griffith, T., 1993. A second generation user interface design environment: the model and the runtime architecture. In: Proceedings InterCHI'93, April 1993, pp. 375-382.
Sukaviriya, P., Kovacevic, S., Foley, J.D., Myers, B., Olsen, D., Schneider-Hufschmidt, M., 1994. Model-based user interfaces, what are they and why should we care? In: Proceedings UIST'94, November 1994, pp. 133-135.

Sun Microsystems, 1998. PersonalJava ${ }^{\mathrm{TM}}$ Technology—White Paper, August.

Sun Microsystems, 2000. Java ${ }^{\mathrm{TM}} 2$ Platform, Standard Edition White Paper, June 2000.

Sun Microsystems, 2000. Java ${ }^{\mathrm{TM}} 2$ Platform Micro Edition(J2ME $\left.{ }^{\mathrm{TM}}\right)$ Technology for Creating Mobile Devices, White Paper, May 2000.

Szekely, P., 1996. Retrospective and challenges for model-based interface development. In: Proceedings of 3rd Int. Workshop on Computer-Aided Design of User Interfaces CADUI'96, June 1996, pp. xxi-xliv.

Szekely, P., Luo, P., Neches, R., 1992. Facilitating the exploration of interface design alternatives: the HUMANOID model of interface design. In: Proceedings CHI'92, May 1992, pp. 507-514.

Szekely, P., Luo, P., Neches, R., 1993. Beyond interface builders: model-based interface tools. In: Proceedings InterCHI '93 April 1993, pp. 383-390.

Vanderdonckt, J., 1995. Knowledge-based systems for automated user interface generation: the trident experience. Technical Report RP95-010, Fac. Univ. de N-D de la Paix, Inst. D’Informatique, Namur.

White, J., et al., 1997. System and Method for Distributed Computation Based upon the Movement, Execution, and Interaction of Processes in a Network, US patent no. 5603031, February 1997.

Wiebus, S., Connect4 Applet. Available from <http://www.physics.adelaide.edu.au/ swright/java_apps/Connect4/>.

Weicha, C., Bennett, W., Boies, S., Gould, J., Green, S., 1990. ITS: a tool for rapidly developing interactive applications. ACM Transactions on Information Systems 8 (3), 204-236.

W3C, 2000. Scalable Vector Graphics (SVG) 1.0 Specification, November 2000. Available from <http://www.w3.org/TR/SVG/>.
1403 